\section{Prototipo de un colector solar parabólico para la generación de energía eléctrica}

\section{Prototype of a parabolic solar collector for production of electrical energy}

\author{
Gerardo Rivera Barrera*, Faiver Tovar Galindo** \\ * Corporación Universitaria del Huila corhuila. McS. Docente de tiempo completo, Facultad de Ingeniería \\ ** Maestría en Física McS. Corporación Universitaria del Huila Corhuila. Docente de tiempo completo. \\ Facultad de Ingeniería
}

\section{Resumen}

El objetivo del proyecto de investigación fue el diseño de un prototipo de un colector solar parabólico reflector de seguimiento real que suministre energía térmica suficiente que puede ser utilizada en la industria o en la demanda energética de los hogares. Para la realización de este proyecto se inició con el estudio de los antecedentes temáticos de colectores solares, su distribución, variación y componentes de la radiación solar terrestre. Igualmente se revisó la documentación teórica sobre la intensidad de la radiación solar en Colombia y la distribución de energía disponible en la región, posteriormente se inicio el diseño del colector parabólico de manera simulada que nos permitirá definir las partes, el tamaño y la intensidad de radiación térmica generada por este y luego poder continuar con la construcción del colector solar. Con el proyecto se logró verificar la efectividad del colector solar y la capacidad que posee de suministrar energía térmica suficiente para ser conectada a un generador eléctrico para el abastecimiento de la industria o de un hogar promedio en nuestra región.

Palabras clave: Celdas fotovoltaicas, colector solar, modelaje, simulaciones, sistemas numéricos.

\begin{abstract}
The purpose of this research project was to design a prototype of a parabolic solar collector real tracking reflector that provides sufficient thermal energy to be used either by industry or home energy demand. The project started by studying records of solar collectors, their distribution, variation and terrestrial solar radiation components. Likewise, theoretic documents on solar radiation intensity in Colombia, and distribution of available energy in the region were reviewed. Subsequently, the design of a simulated parabolic collector was started, which will lead to define parts, size, and thermal radiation intensity produced by this collector, and then continue with construction of the solar collector. The project led to verify the solar collector effectiveness, and its ability to provide sufficient thermal energy to be connected to an electrical generator, to supply either the industry, or an average home in our region.
\end{abstract}

Key words: Photovoltaic cells, solar collector, modeling, simulations, numerical systems.
Recibido: 12/04/2016

Revisado: $15 / 05 / 2016$

Aceptado:23/12/2016

Correspondencia de autor:

gerardo.rivera@corhuila.edu.co

faiber.tovar@corhuila.edu.co

(C) 2016 Universidad La Gran Colombia. Este es un artículo de acceso abierto, distribuido bajo los términos de la licencia Creative Commons Attribution License, que permite el uso ilimitado, distribución y reproducción en cualquier medio, siempre que el autor original y la fuente se acrediten.

\section{Cómo citar:}

Rivera, G., Tovar, F. (2016) Prototipo de un colector solar parabólico para la generación de energía eléctrica.

UGCiencia 22, 149-158. 


\section{Introducción}

En Colombia la preocupación por el ahorro energético ha llevado al gobierno nacional, al sector privado, a los grupos de investigación de altas energías y energías renovables a pensar en soluciones, económicas, ambientales y científicamente posibles en busca de fuentes alternas de energías renovables limpias y seguras, cuya disponibilidad es visible como en el caso de la energía eólica, biomasa, energía solar, entre otras.

La energía solar es en esencia el estudio más importante de las energías renovables, ya que se han creado en países desarrollados incentivos de carácter económico e investigativo para el desarrollo de nuevas tecnologías e infraestructuras que permitan el aprovechamiento de la energía solar, luego su principal ventaja con la que cuenta la energía solar es que se trata de un recurso inagotable que está al alcance de todos. La eficiencia energética y la búsqueda de nuevas fuentes de energía han llevado a investigar aplicaciones de la energía solar para generación de energía eléctrica.

La energía solar posee numerosas aplicaciones donde puede ser utilizada. Estas se clasifican en tres grupos: generación térmica, de trabajo y de frío. El primer grupo contiene la generación de calor para calefacción, agua caliente sanitaria, desecación y refrigeración. En el segundo grupo se puede dar de forma térmica y de forma fotovoltaica y el tercer grupo se produce por medio de máquinas de absorción.

Finalmente, para utilizar eficazmente este recurso energético, se diseñará un colector solar parabólico para la generación de energía eléctrica a través de celdas fotovoltaicas y poder obtener un óptimo rendimiento energético a partir de su diseño y aplicación.

\section{Perspectiva teórica}

El sol es una estrella que se encuentra a una temperatura media de $5.500{ }^{\circ} \mathrm{C}$, en cuyo interior tienen lugar una serie de reacciones que producen una pérdida de masa que se transforma en energía. Esta energía se transmite al exterior mediante la denominada radiación solar, que es una cantidad de energía procedente del Sol que se recibe en una superficie y en un tiempo determinado. Su intensidad depende de la altura solar (latitud, fecha y hora del día), condiciones atmosféricas y altura sobre el nivel del mar. Los siguientes trabajos de investigación son referencias de estudios realizados sobre la formulación teórica y el diseño de un colector solar parabólico con diferentes aplicaciones.

En el año 2007, C. Polo y Col, desarrollaron una evaluación de un concentrador solar cilíndrico parabólico, que consiste en un sistema, que toma la energía solar por medio de un tubo absorbente, el cual, está constituido por una envoltura de vidrio para el aprovechamiento de la radiación solar. Este concentrador fue elaborado por los estudiantes de la especialidad de energías renovables de la escuela academia profesional de física, aplicada de la facultad de ciencias en las instalaciones del centro de energías renovables de Tacna, de la Facultad de Ciencias de la Universidad Nacional Jorge Basadre Grohmann de Tacna, por lo cual, se evalúa aspectos como: determinación del área eficiente de la superficie reflectante, pérdidas de calor debido al ángulo de inclinación del concentrador, determinación de la eficiencia óptica, determinación del coeficiente global de pérdidas y la determinación de la eficiencia térmica.

Posteriormente, L. Vidríales Escobar (2007), mostró que existen diferentes formas para la obtención de energía renovable, concluyendo que la forma más viable para la economía de las personas y una mejor conservación de los recursos ecológicos, es la captación de la 
energía por medio de la radiación solar, por esta razón se desarrolló el diseño, construcción y evaluación de un sistema de concentrador solar de un canal parabólico (CCP),el cual, su receptor, es un modelo de tubo absorbedor, UVAC (Colector Universal de Vacío, Solel) 2003, es la tercera generación de los tubos de vacío. Para la producción directa de vapor de agua con baja entalpía. Es decir, generar vapor a $120^{\circ} \mathrm{C}$ y con una presión absoluta cercana a 1.7 Bar a partir de energía solar.

J. Quinteros Grijalva en el 2008, realizó el estudio sobre la búsqueda de nuevas fuentes de energía por medio de radiación solar, donde se desarrolló aplicabilidad de colectores solares parabólicos, junto con un ciclo de vapor para generación de energía ó la utilización de un motor Stirling, donde pueden ser alternativas válidas de implementación para generación distribuida, sobre todo en regiones de Chile donde existe una alta radiación solar. Por lo cual se diseña y se construye este sistema para seguir la forma autónoma al sol, en la Universidad de Chile Facultad de Ciencias Físicas y Matemáticas Departamento de Ingeniería Eléctrica.

Además en el 2008 Venegas Reyes, realizo la construcción de un sistema para la generación de energía eléctrica y el almacenamiento de calor de proceso por medio de un concentrador solar de foco puntual con ángulo de apertura de $90^{\circ}$, denominado cospaa-90. Este tipo de concentrador solar, está conformado por una antena parabólica de telecomunicaciones de aluminio y se debe llevar a cabo el adecuado sistema de control de seguimiento del Sol para así, obtener un gran porcentaje de la radiación solar.

Los avances de un prototipo de un colector solar expuesto por Montúfar y Col., 2009, muestran que en la sección de óptica se especifican los avances para obtener una superficie con reflectancia de $94 \%$ que concentre aproximadamente $10 \mathrm{~kW}$ de energía, en la sección termodinámica se han definido los materiales y se han elaborado diagramas de tubería e instrumentación correspondientes al colector del disco parabólico, en el área de control se tienen las especificaciones para los motores y el código para el seguimiento del sol.

Pinedo y Col., 2010, desarrollaron el análisis, diseño y construcción de un colector parabólico, donde se desarrolló mejoras como la incorporación de espejos reflectantes y foto sensores que ayudan al seguimiento de la luz del sol, por lo tanto, se produjo los aumentos de la eficacia y eficiencia del sistema como tal. Donde le puede dar aplicabilidad en sistemas domésticos de calefacción, mejoramiento de producción anaeróbica de fermentación, hasta centrales termo solares para la generación de energía eléctrica. En la parte final, se presentan propuestas para la mejora de este sistema en función al estudio teórico realizado.

En otro proyecto de investigación se realizó la construcción y el análisis de un prototipo de un colector solar para la generación de energía eléctrica a partir del aprovechamiento de la energía térmica (solar). Martorelli y Col., 2011, primero desarrollaron un prototipo experimental del concentrador óptico parabólico de 1,5 m de diámetro, que se ha sido diseñado y construido, a fin de analizar y evaluar ciertos aspectos de investigación puntuales, lo cual nos llevará al diseño del prototipo final del concentrador óptico parabólico (COP) de $7 \mathrm{~m}$, en la cual el proceso se desarrolló en la Universidad Nacional de La Plata a través del Laboratorio de Óptica de Calibraciones y ensayos. Facultad de Ciencias Astronómicas y Geofísicas.

Para este tipo de prototipo experimental existen básicamente dos tipos de colectores solares: los no concentradores y los concentradores.

Un colector plano típico es cuando la radiación solar pasa a través del vidrio cobertor e incide sobre la superficie absorbente, una gran cantidad 
de esta energía es transferida directamente al líquido dentro de los tubos (comúnmente agua), y este es luego almacenado u ocupado en calefacción. Los colectores parabólicos compuestos tienen la capacidad de reflejar al foco lineal toda la radiación incidente que captan. Usando múltiples reflexiones internas, cualquier radiación solar que entre al colector dentro de su ángulo de aceptancia $\left(0^{\mathrm{C}}\right)$, llega a la superficie absorbente que está en el fondo del colector. Los colectores concentradores presentan ciertas ventajas con respecto al colector plano convencional. Las principales son:

a. El fluido de trabajo llega a temperaturas mayores en un colector concentrador, tomando la misma significa que se puede lograr una mayor eficiencia termodinámica.

b. Dado que la superficie absorbente es pequeña (con respecto al arca total del colector), ocupar superficies de absorción selectivas y ocupar zonas de vacío en esta para disminuir pérdidas (y por lo tanto la efectividad del colector) son alternativas económicamente viables. También ofrecen desventajas comparativas. Los principales son:

1. Los colectores concentradores solo utilizan la radiación directa y no la difusa.

2. Es necesario implementar un sistema de seguimiento solar con los colectores.

3. El reflectante del colector puede perder sus propiedades con el tiempo y requiere mantenimiento.

Los colectores que entran en esta categoría son: colectores cilindro parabólicos, disco parabólico y helióstato. Los colectores cilindro parabólicos tienen una característica importante, que pueden lograr temperaturas hasta los $400^{\circ} \mathrm{C}$ con una buena eficiencia termodinámica. Tienen aplicaciones tanto en generación de energía eléctrica como en calentamiento de agua, pudiendo para esta última aplicación ser construidos con materiales ligeros y baratos, lo cual disminuye el costo de inversión inicial y facilita el seguimiento solar. Estos colectores se construyen doblando una lámina de material reflectante con forma parabólica. Un tubo metálico de color negro, recubierto con un tubo de vidrio para disminuir las pérdidas de calor, se debe colocar a lo largo de la línea focal como receptor.

Un disco o colector parabólico se encuentra enfrente al sol, la radiación incidente se concentra en su foco. Por lo anterior, debe seguir al sol en sus dos grados de libertad (altitud y azimut).

El receptor colocado en el foco, absorbe la radiación solar y la traspasa en forma de calor a un fluido circulante. La energía calórica presente en el fluido puede ser convertida a electricidad mediante el uso de un motor generador dispuesto en el foco o puede ser transportada a través de cañerías a un receptor central y servir para diversos propósitos. La temperatura en el receptor puede sobrepasar los $1500^{\circ} \mathrm{C}$. Los colectores parabólicos tienen ventajas importantes con respecto a otros prototipos diseñados en las literaturas mostradas para esto tenemos que [3-9]: 1- Siempre están apuntando al sol, son un sistema de concentración solar más eficiente. 2- Tienen concentraciones entre 600 - $2000{ }^{\circ} \mathrm{C}$. 3- El colector como modulo se puede ocupar de manera independiente o ser parte de un campo de colectores.

Un helióstato, puede obtener altos niveles de radiación de energía solar, para esto es necesario disponer de varios espejos planos dispuestos en un campo con seguimiento solar de altitud-azimut y reflectando la radiación incidente en cada uno de ellos a un foco receptor común. Generalmente, en el receptor se genera vapor de agua a alta presión y temperatura. Esta agua se lleva mediante cañerías a un receptor central donde se puede almacenar y ocupar para generar energía eléctrica. 
Se pueden obtener concentraciones entre 300 y 1500 , teniendo el foco un flujo radiactivo entre 200 y $1000 \quad\left[\mathrm{~kW}=\mathrm{m}^{2}\right]$, obteniéndose temperaturas por sobre los $1500^{\circ} \mathrm{C}$.

Posteriormente con el fin de maximizar la energía solar que llega al receptor, un sistema de seguimiento solar debe ser desarrollado. En un colector parabólico es muy importante, ya que los rayos que inciden de manera paralela a la línea focal inciden en el foco, por lo tanto, es necesario estar apuntando al sol en todo momento. Este sistema de seguimiento debe ser confiable, capaz de seguir al sol con un cierto nivel de certeza (que depende de la aplicación que se requiera), capaz de regresar al colector de su posición en el ocaso a su posición de espera del sol en el amanecer y también seguir al sol aun cuando haya existencia de nubes en forma intermitente. Adicionalmente, el sistema de seguimiento también puede ser usado para proteger al colector ante la presencia de condiciones adversas de trabajo, como cambios climáticos, falla en el flujo del fluido de enfriamiento.

En este proyecto de investigación se presentó una modelización matemática, en la aplicación Matlab, para realizar el diseño preliminar de un campo solar de heliostatos de una central termoeléctrica, basada en la tecnología de concentración solar con receptor central de torre. Se estudió el principal recurso energético, el Sol, cómo llega su energía a la Tierra en forma de radiación, y los distintos procesos que determinan la distribución del recurso solar por el planeta. Luego se detallarán las principales tecnologías existentes basadas en técnicas de concentración solar, que aprovechan este recurso energético para la producción de electricidad. Después, se comenzará a implementar un programa matemático, que realizará el diseño óptimo inicial de un campo solar, a partir de un algoritmo y parámetros básicos de diseño.
Actualmente, debido al desarrollo de la electrónica, el seguimiento alt-azimutal es el más utilizado. En este tipo de seguimiento, se utilizan dos motores (uno por eje de giro) los cuales son controlados electrónicamente a través de sensores (por ejemplo fotodiodos) que detectan la radiación solar incidente. Cuando la radiación sobre un sensor disminuye, entonces se da la orden de giro en el eje correspondiente

La utilización de energía solar es el mejor ejemplo de una práctica sostenible: se encuentra asociada con reducidas externalidades ambientales, hace uso de un recurso $100 \%$ renovable y genera importantes ahorros para sus usuarios (la inversión inicial es superior, pero los costos de operación son significativamente más bajos). Este tipo de tecnologías se ajusta tanto a aplicaciones urbanas (semáforos, iluminación, cámaras de seguridad, etc.).

La intensidad de la radiación solar en la región Andina colombiana muestra que las zonas de los valles del Cauca y Magdalena poseen el mayor potencial de esta región, y a medida que se asciende hacia las cimas de las cordilleras ese potencial va disminuyendo gradualmente, con excepción de algunos núcleos localizados en zonas llamadas altiplanos, donde se observa un potencial solar mayor comparado con el de las laderas. En las regiones costeras: atlántica y pacífica, los resultados de la evaluación del recurso solar del país muestran en la región noreste de la costa atlántica (La Guajira) un potencial solar promedio diario entre $5.0 \mathrm{y}$ $6.0 \mathrm{kWh} / \mathrm{m}^{2}$, el mayor del país. Este valor va disminuyendo gradualmente en dirección sur-oeste hacia la costa pacífica, donde se presenta el menor potencial solar del país, con valores menores de $3.5 \mathrm{kWh} / \mathrm{m}^{2}$, aunque posee una gran zona con valores entre 4.0 y 4.5 [11]. Las regiones de la Orinoquia y Amazonía, que comprenden las planicies de los Llanos Orientales y zonas de las selvas colombianas, 
presentan una variación ascendente de la radiación solar en sentido suroeste-noreste, verificándose valores asimilables a los de La Guajira en el noreste (Puerto Carreño).

F. Xie, y Col (2011) desarrollaron simulaciones y análisis de sistemas de seguimiento de control de equipo basado en geometría solar para lentes de Fresnel Photovoltics Concentration. Es un sistema construido con dos ejes accionado por el motor con la retroalimentación del sensor de ángulo, donde estos determinan posiciones solares que son calculadas a partir de algoritmo solar.

En otro trabajo de investigación se diseñó un sistema que se posiciona de forma automática en el punto de máxima irradiación solar a través de un algoritmo que calcula los ángulos de azimut y de elevación necesario. Los ángulos que se calcularon y los parámetros evaluados permitieron posicionar los motores de cada eje del sistema, estos son enviados a un microcontrolador ATMega16 mediante el puerto serie de una PC.

R. M. González García (2014), utilizo la geometría para reflejar los rayos solares hacia el receptor, es decir la geometría del plato parabólico. Esta propuesta de investigacion se realizó mediante la ayuda del software de análisis de trazado de rayos SolTrace.con la geometría óptima de la superficie concentradora de este sistema, se diseñó el disco parabólico, esto se realizó con la ayuda del software de diseño mecánico NX6. En paralelo se eligió la superficie reflejante del disco parabólico. Con el diseño y los planos de fabricación se procedió a la construcción del disco parabólico de $2 \mathrm{~m}$ de diámetro. Se eligió aluminio como base de la estructura por su buena relación resistenciapeso, y una película reflejante Reflectech, con $95 \%$ de reflectividad. Debido a que la manufactura del ángulo de apertura exacto $\left(45^{\circ}\right)$ implicó un proceso complejo y caro.
Para lograr la optimización de la energía obtenida en una instalación fotovoltaica existen dos métodos. El primero consiste en mejorar los componentes internos de un panel fotovoltaico de manera que su rendimiento aumente. El segundo seria aumentar la cantidad de radiación solar recibida por el panel. En esta propuesta de investigación se enfocó en que el panel recibiera la mayor cantidad de radiación solar, buscando que el área del panel fotovoltaico permanezca en posición perpendicular a la radiación lumínica de la fuente de luz, lo cual se logra mediante un algoritmo programado en un microcontrolador Motorola.

\section{Materiales y métodos}

Para el diseño del prototipo se inició con la recopilación de los antecedentes generales sobre la distribución, variación y componentes de la radiación solar terrestre disponible anual en nuestra región. También se revisó la distribución de la intensidad de la radiación solar en Colombia.

Posteriormente se inició con el diseño del prototipo para el colector parabólico reflector de manera teórico-simulado que nos permitió definir las partes, el tamaño y la intensidad de radiación térmica que puede generar este prototipo, para luego definir la construcción del colector parabólico de acuerdo con los resultados simulados obtenidos.

Para el desarrollo de la propuesta se definieron las siguientes actividades:

Fase antecedentes generales

- Radiación terrestre.

- Sistemas de colectores parabólicos.

- Seguimiento solar en dos ejes.

- Determinación de la distribución de la radiación solar disponible en Colombia.

- Determinación de la distribución de la radiación solar disponible en la región. 
Fase de simulación

- Simulación mecánica y cálculo numérico. Modelación.

- Simulación del colector parabólico.

- Diseño, incidencia solar y punto focal.

Fase de diseño y prototipo

- Diseño del prototipo del reflector solar parabólico.

- Configuración del colector solar parabólico.

- Determinación del tamaño del colector parabólico.

- Diseño del sistema de almacenamiento.

Fase de evaluación

Durante la fase de evaluación, se verificará la efectividad del colector solar de acuerdo con los resultados obtenidos en las simulaciones numéricas.

\section{Discusión de resultados}

Se realizó el diseño y simulación del colector solar parabólico utilizando software de modelación y de análisis numérico de uso comercial. Los softwares utilizados fueron el Solid Word y el Matlab, con los cuales fue posible parametrizar las variables requeridas para su diseño y construcción de acuerdo con las estimaciones tomadas, el diámetro de curvatura, los cálculos numéricos y los resultados teóricos dados. Para este prototipo se recomienda la utilización del acero inoxidable galvanizado siendo un material de alta reflexión, duradero (variaciones climáticas y exposiciones a altas temperaturas) y de bajo mantenimiento. También es un excelente reflector solar con alta incidencia y concentración tanto para colectores parabólicos como para reflectores Offset.

De acuerdo con los resultados obtenidos, tanto teóricos como los simulados, tenemos que: De manera teórica simulada obtuvimos que $\mathrm{D}=$ $1500 \mathrm{~mm}, \mathrm{P}=281 \mathrm{~mm}$ entonces, la distancia focal calculada fue:

$$
f=\frac{(1500)^{2}}{16 *(281)} \mathrm{mm}=500.4445 \mathrm{~mm}
$$

Donde

$$
f / D=\frac{500.4445}{1500}=0.334
$$

Luego el ángulo iluminación:

$$
\propto=2 \operatorname{arcotag}\left(\frac{1500 / 2}{500.445-281}\right)=147.38^{\circ}
$$

Luego utilizando el simulador verificamos que:

Diámetro Lineal: $\quad 1630,29$

Diámetro: $\quad 1500.00$

Profundidad: $\quad 281.00$

Distancia focal: $\quad 500,44$

Long. circunf: $\quad 4712.39$

Volumen parab: 248283994.40

Área parab.

1767146.00

Relacion F/D: $\quad 0,33$

Relacion D/F: $\quad 3$

Ang. a Iluminar: $\quad 147.38^{\circ}$

Ang.de Ilumin.del feed: $90^{\circ}$

Con esto podemos verificar que los porciento de diferencia de las distancias focales de los datos simulados y del método numérico son bastantes similares permitiéndonos verificarlos las aproximaciones numéricas para el diseño del colector. Para el proceso de construcción del prototipo se debe elabora un panel circular cuyo diámetro será de $123.87 \mathrm{~mm}$. Permitiendo recubrir dicho panel de materiales reflectores que nos permitirán la mayor efectividad de la generación de energía térmica, permitiéndonos la utilización de celdas fotovoltaicas que serán las encargadas de la trasformación de energía solar a energía eléctrica. 
En la figura 1 se muestra el diseño del colector solar bajo la toma de datos del sistema numérico, encontrando una distancia focal de 500,44 $\mathrm{mm}$ una profundidad de plato de $281 \mathrm{~mm}$, un diámetro lineal de1630,31 mm, una longitud de circunferencia de 4712,39 $\mathrm{mm}$ y un área de parábola de $1767146 \mathrm{~mm}$.

Figura 1. Colector solar de manera teórico bajo el simulador. Fuente propia.

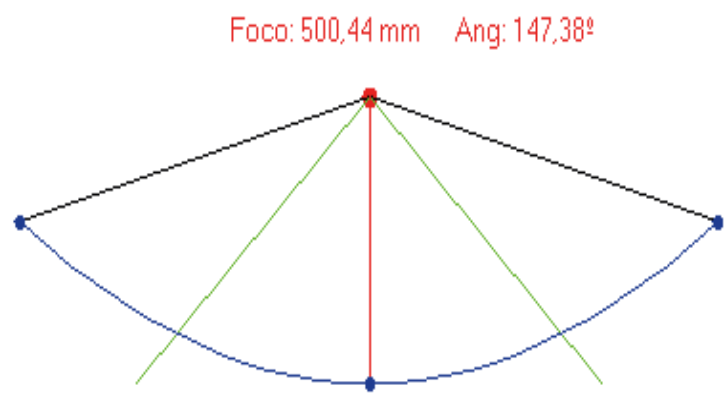

La figura 2 y 3 hace referencia a los diseños asistidos por computadora del modelado mecánico del colector solar parabólico en 3D, de acuerdo a los resultados obtenidos por las simulaciones numéricas, obteniendo el colector ideal de acuerdo a la evaluación de los parámetros seleccionados.

Figura 2. Diseño del colector solar parabólico asistido por computadora con el SolidWord. Fuente Propia

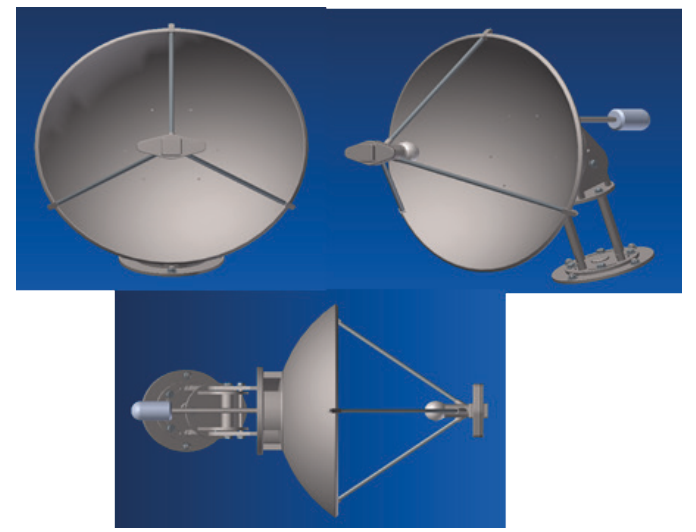

Figura 3. Colector solar parabólico de diámetro de $1,5 \mathrm{~m}$ de longitud.
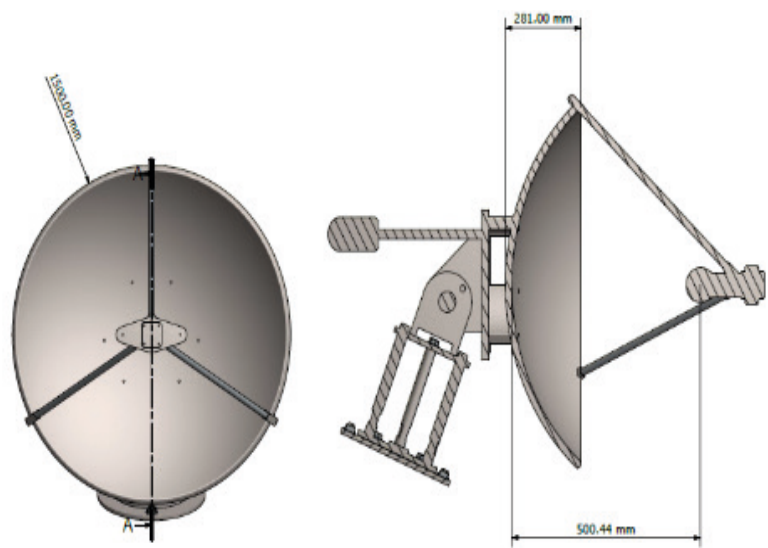

Diseño elaborado SolidWork. Fuente propia.

\section{Conclusiones}

En nuestro departamento del Huila contamos con una muy buena fuente de energía solar durante todo el año, la cual hace propicio el estudio y desarrollo de un colector solar parabólico reflector de seguimiento real para la generación de energía eléctrica utilizando un sistema fotovoltaico o sistemas térmicos pertinentes que nos permitan la transformación de esta energía captada por el modelo.

En el proyecto se logró diseñar el prototipo del colector solar parabólico reflector de acuerdo con las especificaciones teóricas y simuladas tomando como mejores resultados el reflector de un diámetro de 1,5 metros de longitud donde se verificó la mayor concentración de energía térmica, para estos procesos su utilizo el Solid Word como software especializado en diseño y el Matlab como software especializado en el método numérico. Logrando establecer los parámetros del prototipo y fundamentar el diseño del rotor que permitirá el seguimiento real del colector de acuerdo con el movimiento rotacional del sol en nuestra región. 


\section{Recomendación}

Se recomienda la construcción del colector solar reflector con seguimiento real, con los datos que se obtuvieron en el análisis de la simulación y métodos numéricos, para verificar su funcionalidad y poder emplear celdas fotovoltaicas con el objetivo de verificar su óptimo rendimiento energético y confirmar su aplicación de este para el uso industrial o para el servicio doméstico habitacional en nuestra región proporcionando un recurso de energía limpia y sin mayor costo.

\section{Referencias bibliográficas}

Polo, C., Sacari, E.J. (2007). Evaluación experimental de un concentrador solar cilíndrico parabólico (CCP). Universidad Nacional Jorge Basadre Grohmann de Tacna.

Vidríales, L.G. (2007). Colector de canal parabólico para la generación directa de vapor para calor de proceso. Universidad Nacional Autónoma de México. Tesis de Maestría.

Quinteros Grijalva, J. (2008). Estudio teórico y experimental de colector solar parabólico para generación de energía eléctrica. Universidad de Chile. Trabajo de grado.

Venegas, E. (2008). Sistema para generación y almacenamiento de calor de proceso mediante un concentrador solar de foco puntual (cospaa-90). Universidad Nacional Autónoma de México

Araujo, A. P, \& Campos, D. A. (2011). Análisis teórico-experimental del colector solar cilíndrico parabólico automatizado con espejos reflectantes Universidad del Norte sede Trujillo. Tesis de Maestría.
Martorelli, L. C, Biotti, M. A., Haag, G, Costanzo, M \& Winsch, C. (2011). Análisis y evaluación de variables ópticas en el desarrollo de concentradores solares ópticos para la generación de energía eléctrica. Universidad Nacional de La Plata.

Escobar, L. G. V. (2007). Colector de Canal Parabólico para la Generación Directa de Vapor para Calor de Proceso (Doctoral dissertation, Centro de investigación de energía. Universidad Nacional Autónoma de México)

Gómez, C. (2011). Diseño del campo de heliostatos para torres solares de receptor central. Director de proyecto: DJ Santana. Proyecto final de carrera, Universidad Carlos III de Madrid.

Rodríguez, H., \& González, F. (1992). Manual de radiación solar en Colombia: radiación sobre superficies horizontales. Serie sobre Energía. Departamento de Física de la Universidad Nacional de Colombia, Bogotá.

Xie, F., \& Zhang, G. (2011, August). Simulation and Analysis of Computer Control Tracking System Based on Solar Geometric for Fresnel Lenses Concentration Photovoltics. In Intelligent Human-Machine Systems and Cybernetics (IHMSC), 2011 International Conference on (Vol. 1, pp. 101-104). IEEE.

Fuentevilla, J. S., Ávalos, M. J. L. B., \& García, D. A. A. (2012). Diseño y construcción de un sistema de seguimiento fotovoltaico. Universidad Tecnológica de la Mixteca. 
R. M. González, (2014). Diseño mecánico del disco parabólico de un concentrador solar y la caracterización térmica del receptor. Universidad Nacional Autónoma de México.

Mejía, A. E, Londoño, M. H \& Osorio, J. C. (2010). Diseño e implementación de un seguidor solar para la optimización de un sistema fotovoltaico. Scientia et technica, 1(44), 245-250. 